\title{
Reduced-Complexity Detection and Phase Synchronization of CPM Signals
}

\author{
Giulio Colavolpe, Student Member, IEEE, and Riccardo Raheli, Member, IEEE
}

\begin{abstract}
Based on a recent extension of Laurent decomposition of continuous phase modulation (CPM) signals into a sum of linearly modulated components, we derive a class of reducedcomplexity maximum-likelihood (ML) coherent detection and closed-loop phase synchronization schemes.

The complexity of the resulting detection schemes, expressed in terms of the number of matched filters and states of a Viterbi algorithm, is significantly reduced with respect to that of optimal coherent receivers with negligible performance loss. This result extends a known one valid for the binary case to multilevel CPM. The proposed synchronization schemes do not require an increased number of matched filters, and are perfectly suitable to be used in conjunction with these receivers.

Based on the phase-locked loop (PLL) equivalent linear model, a method for optimizing the parameters of digital second-order PLL's is presented. Numerical examples, based on theoretical analysis and computer simulation, are provided for two specific formats in the CPM class: tamed frequency modulation (TFM) and a quaternary raised-cosine (RC) modulation.
\end{abstract}

Index Terms - Continuous phase modulation, maximumlikelihood estimation, phase-locked loops.

\section{INTRODUCTION}

C PM $M$-ary signals are attractive for their good power and spectral efficiency within the class of constant envelope signals, but often require a maximum likelihood sequence detector implemented using the Viterbi algorithm [1]. The optimal receiver consists of a bank of $2 M^{L}$ matched filters, $L$ being the duration in symbol intervals of the so-called frequency pulse, followed by a Viterbi processor which searches a trellis diagram with $p M^{L-1}$ states, where $p$ is the number of phase states. As a consequence, multilevel schemes with large $L$, which offer a large efficiency in terms of power and spectrum, require rather complex receivers.

Several authors have approached the problem of reducing the complexity of the optimal receiver by means of a search on a trellis with a reduced number of states [2] or through a "reduced search" on the entire original trellis [3]. In [4], the authors propose a simplified signal representation, which results in a reduction of the number of matched filters, and techniques for reducing the state complexity of the trellis

Paper approved by O. Andrisano, the Editor for Modulation for Fading Channels of the IEEE Communications Society. Manuscript received October 15, 1996; revised January 11, 1997 and April 8, 1997. This work was performed in part within a research cooperation between the Dipartimento di Ingegneria dell'Informazione, Università di Parma, Italy, and Italtel S.p.A., Milano, Italy. This paper was presented in part at the IEEE International Conference on Communications, Montréal, P.Q., Canada, June 1997.

The authors are with the Dipartimento di Ingegneria dell'Informazione, Università di Parma, 43100 Parma, Italy.

Publisher Item Identifier S 0090-6778(97)06620-8. diagram. In [5], they also face the problem of phase and timing synchronization by decision-directed ML estimation exploiting the specific signal representation.

In terms of complexity reduction, a more efficient signal representation is adopted in this paper. By means of Laurent representation [6], recently extended by Mengali and Morelli to the $M$-ary case [7], CPM signals may be exactly or approximately decomposed into a sum of linearly modulated components. Laurent representation has been used by Kaleh [8] to significantly reduce the size of the filter bank and the number of trellis states in coherent detectors for binary CPM. Using the extended Laurent representation [7], this result is confirmed to hold for multilevel CPM signals. Similarly to the binary case [8], for $M$-ary CPM signals, the effective number of matched filters and states of the Viterbi processor is quite limited. As an example, for a quaternary $2 \mathrm{RC}$ modulation with index $h=0.25$ (i.e., the frequency pulse is $\mathrm{RC}$ with duration $L=2$ ), a practically optimal receiver is composed of only 4 matched filters and a Viterbi processor with 4 states, whereas the optimal receiver requires 32 filters and 16 states. An alternative derivation of Laurent representation in the special case of a binary CPM with modulation index $h=0.5$ and its possible use for obtaining simplified symbol-by-symbol receivers were presented in [9]. An important characteristic of Laurent representation is the fact that it allows one to achieve a simultaneous reduction in the number of matched filters and trellis states. These complexity reduction tasks are treated separately in [4], with the result that a greater effort is required both at the conceptual and receiver design level.

In synchronization applications, Laurent representation has been used to derive a class of non data-aided frequency detectors [10]. By using the extended Laurent representation, we derive a class of decision-directed reduced-complexity ML closed-loop phase synchronization schemes for $M$-ary CPM signals, which are particularly suitable to be employed in conjunction with optimal or reduced-complexity receivers based on the same signal representation. It is shown that the same filters necessary for coherent detection are also sufficient for synchronization purposes. A carrier recovery algorithm for binary CPM signals with $h=0.5$ [11], similar to a special case of the synchronization schemes proposed in this paper, was brought to the authors' attention during the review process.

The proposed synchronization schemes have been analyzed in terms of acquisition time, robustness to phase jitter, mean time between phase slips, and transient behavior. A method to optimize the tracking properties of the proposed synchronization schemes is proposed, which takes into account the 
possible presence of oscillator instabilities modeled as phasecontinuous step-like instantaneous frequency variations [12], [13]. Computer simulation has also been used to confirm and extend the theoretical analysis.

The paper is organized as follows. In the next section, we review the signal model based on the extended Laurent representation. This signal model is used in Section III to realize reduced-complexity coherent detectors and to derive a general ML synchronization structure. In Section IV, we analyze the proposed synchronization schemes, and provide a loop optimization algorithm. In Section V, we present numerical results. Conclusions are drawn in Section VI.

\section{Signal Model}

The complex envelope of CPM signals has the form [1], [4], [14]

$$
s(t, \boldsymbol{\alpha})=\sqrt{\frac{2 E_{S}}{T}} e^{j \Phi(t, \boldsymbol{\alpha})}
$$

in which $E_{S}$ is the energy per information symbol of the bandpass received signal, $T$ is the symbol interval, and $\Phi(t, \boldsymbol{\alpha})$ represents the information-bearing phase function

$$
\Phi(t, \boldsymbol{\alpha})=2 \pi h \sum_{n=0}^{\infty} \alpha_{n} q(t-n T)
$$

where $h=k / p$ is the modulation index ( $k$ and $p$ are relatively prime integers), the information symbols $\left\{\alpha_{n}\right\}_{n=0}^{\infty}$ are independent and take on values in the $M$-ary alphabet $\{ \pm 1, \pm 3, \cdots, \pm(M-1)\}$ with equal probability, and $\alpha \triangleq\left(\alpha_{0}, \alpha_{1}, \cdots, \alpha_{n}, \cdots\right)$ represents the information sequence. The function $q(t)$ is the phase-smoothing response which describes how the phase evolves with time, and is assumed to satisfy the following normalization condition

$$
q(t)= \begin{cases}0, & \text { for } t \leq 0 \\ 1 / 2, & \text { for } t \geq L T\end{cases}
$$

in which $L$ is a positive integer and $L T$ is the duration of the frequency pulse $g(t)$ defined by

$$
g(t) \triangleq \frac{d q(t)}{d t} .
$$

Based on Laurent representation [6], extended by Mengali and Morelli [7], the complex envelope of CPM signals may be exactly expressed as

$$
s(t, \alpha)=\sqrt{2 E_{S}} \sum_{k=0}^{Q^{P}} \sum_{n} a_{k, n} h_{k}(t-n T)
$$

where $Q \triangleq 2^{L-1}, P \triangleq \log _{2} M$, and the expressions of the pulses $\left\{h_{k}(t)\right\}$, the symbols $\left\{a_{k, n}\right\}$ as a function of the information symbol sequence $\left\{\alpha_{n}\right\}$, and their correlation sequence $R_{k, l}(m)=E\left\{a_{k, n} a_{l, n+m}^{*}\right\}$ may be found in [7]. By truncating the summation in (5) to the first $K \leq Q^{P}\left(2^{P}-1\right)$ terms, the expression of $s(t, \boldsymbol{\alpha})$ may be approximated as

$$
s(t, \boldsymbol{\alpha}) \simeq \sqrt{2 E_{S}} \sum_{k=0}^{K-1} \sum_{n} a_{k, n} p_{k}(t-n T) .
$$

If $K=Q^{P}\left(2^{P}-1\right)$ and the pulses $p_{k}(t)=h_{k}(t)$, we obviously obtain the exact representation. As shown in [7], most of the signal power is concentrated in the first $2^{P}-1=$ $M-1$ components, associated with the pulses $\left\{h_{k}(t)\right\}$ with $0 \leq k \leq 2^{P}-2$, which are denoted as principal. As a consequence, a value of $K=2^{P}-1$ and the pulses $p_{k}(t)=$ $h_{k}(t)$ may be used in (6) to attain a very good tradeoff between approximation quality and number of signal components. For $K=2^{P}-1$, this approximation may be slightly improved by selecting the pulses $\left\{p_{k}(t)\right\}$ in order to minimize the meansquare error (MSE) with respect to the exact signal. Each of the resulting pulses $p_{k}(t)$ may be expressed as the sum of the corresponding $h_{k}(t)$ plus a combination of delayed versions of nonprincipal pulses [7].

As an example for a quaternary CPM, assuming $K=3$ and decomposing the information symbol $\alpha_{n} \in\{ \pm 1, \pm 3\}$ in two binary symbols $\gamma_{n, 0}$ and $\gamma_{n, 1}$ belonging to the alphabet $\{ \pm 1\}$ as suggested in [7], we may write

$$
\alpha_{n}=2 \gamma_{n, 1}+\gamma_{n, 0}
$$

Assuming that $h$ and $2 h$ are not integers, the symbols of the first three signal components may be expressed as (here we follow the notation in [7])

$$
\begin{aligned}
& a_{0, n}=b_{0, n}^{(0)} b_{0, n}^{(1)} \\
& a_{1, n}=b_{0, n-1}^{(0)} b_{0, n}^{(1)} \\
& a_{2, n}=b_{0, n}^{(0)} b_{0, n-1}^{(1)}
\end{aligned}
$$

with

$$
\begin{aligned}
& b_{0, n}^{(0)}=\exp \left\{j h \pi \sum_{m=0}^{n} \gamma_{m, 0}\right\}=b_{0, n-1}^{(0)} e^{j h \pi \gamma_{n, 0}} \\
& b_{0, n}^{(1)}=\exp \left\{j 2 h \pi \sum_{m=0}^{n} \gamma_{m, 1}\right\}=b_{0, n-1}^{(1)} e^{j 2 h \pi \gamma_{n, 1}}
\end{aligned}
$$

This specific case for $L=2$ will be used in the numerical results.

For binary CPM signals, the representation (5) holds with $P=1$ (Laurent representation). The time pulses $\left\{h_{k}(t)\right\}$, the symbols $\left\{a_{k, n}\right\}$ as a function of the information symbol sequence $\left\{\alpha_{n}\right\}$, and their correlation sequence $R_{k, l}(m)$ may also be found in [6]. Note that the symbols $\left\{a_{k, n}\right\}$ are, in general, correlated even when $\left\{\alpha_{n}\right\}$ are independent. Most of the signal power is carried by the signal component corresponding to pulse $h_{0}(t)$. The duration of this pulse is $(L+1) T$. The next significant pulse is $h_{1}(t)$ whose duration is $(L-1) T$.

In the special case of minimum shift-keying (MSK)-type modulations, i.e., binary CPM with $h=0.5$ (also called generalized MSK), the approximation with the first component is optimal in the MSE sense $\left[p_{0}(t)=h_{0}(t)\right]$ [6]. The symbol sequence $\left\{a_{0, n}\right\}$ corresponding to first signal component is simply related to the information symbol sequence $\left\{\alpha_{n}\right\}$ by

$$
a_{0, n}=j \alpha_{n} a_{0, n-1}, \quad\left\{\begin{array}{l}
a_{0,2 n} \in\{ \pm 1\} \\
a_{0,2 n+1} \in\{ \pm j\} .
\end{array}\right.
$$


For $L \geq 2$, the symbol sequence $\left\{a_{1, n}\right\}$ corresponding to the second signal component is defined by

$$
a_{1, n}=j \alpha_{n} a_{0, n-2}, \quad\left\{\begin{array}{l}
a_{1,2 n} \in\{ \pm j\} \\
a_{1,2 n+1} \in\{ \pm 1\} .
\end{array}\right.
$$

Symbols $a_{k, n}$ and $a_{l, m}$ are uncorrelated for $k \neq l$ and for $n \neq m$, i.e.,

$$
\begin{aligned}
R_{k, l}(n-m) & =E\left\{a_{k, n} a_{l, m}^{*}\right\} \\
& = \begin{cases}1, & \text { for } k=l \text { and } n=m \\
0, & \text { otherwise. }\end{cases}
\end{aligned}
$$

Evidently, the first component is sufficient to carry the binary information symbols; the raison d'être of the other components is to give to the signal its constant envelope.

In this paper, the approximation (6) represents the conceptual basis for the derivation of the detection and phase synchronization schemes. Since most of the signal power is carried by a few signal components, we consider only $K<Q^{P}\left(2^{P}-1\right)$ terms in (6). This approach has been used in [8] to derive reduced-complexity near-optimal receivers for Gaussian-MSK (GMSK) modulation. These receivers consider at most two signal components [those associated with $h_{0}(t)$ and $\left.h_{1}(t)\right]$. The justification for this approach is based on the results reported in [8] which show that a fraction 0.992 of the total signal power is carried by the first signal component, a fraction 0.008 by the second, and only a fraction $2.63 \times 10^{-5}$ by the others.

For TFM (binary CPM with $h=0.5$ ) [15] and quaternary 2RC modulation with $h=0.25$, we observed a similar behavior, i.e., approximations of CPM signals based on the first linear components practically entail negligible degradations. Specifically, the first two and three components may be used to approximate the considered TFM and quaternary CPM formats, respectively. As an example, for the considered quaternary $2 \mathrm{RC}$ modulation, the energy of the first signal component pulse, normalized to the sum of the energy of all pulses, is 0.751 , the second and the third pulses have normalized energy 0.129 each, whereas the fraction of energy of the remaining pulses is $3.6 \times 10^{-4}$. We remark that, unlike the case of MSK-type modulations in which the energy of each pulse corresponds to the fraction of the total signal power carried by the relevant signal component [6], for $2 \mathrm{RC}$ modulation with $h=0.25$ and $M=4$, this is true only approximately due to symbol correlation.

These conclusions on complexity reduction of the receiver structures may also be drawn in applications of Laurent representation to the realization of frequency detectors such as those proposed in [10], in which, for MSK-type signals, no performance benefit is attained taking into account more than two components, and extend to the proposed detection and phase synchronization schemes.

\section{REDUCED-COMPLEXITY COHERENT DETECTION AND PHASE SYNCHRONIZATION}

The complex envelope of the received signal may be modeled, assuming perfect knowledge of symbol timing, as

$$
r(t)=s(t, \alpha) e^{j \theta}+w(t)
$$

where $w(t)$ is a complex-valued Gaussian white noise process with independent components, each with two-sided power spectral density $N_{0}$, and $\theta$ is a constant or slowly varying phase shift introduced by the channel.

Under the assumption that the received signal is observed in the interval $\left(-T_{0} / 2, T_{0} / 2\right)$ and exploiting the constant envelope property of a CPM signal, the relevant likelihood function for both coherent detection and phase synchronization is [1]

$$
\Lambda(\tilde{\theta}, \tilde{\boldsymbol{\alpha}})=\frac{1}{\sqrt{2 E_{S}}} \operatorname{Re}\left\{\int_{-T_{0} / 2}^{T_{0} / 2} r(t) s^{*}(t, \tilde{\boldsymbol{\alpha}}) e^{-j \tilde{\theta}} d t\right\}
$$

where $\tilde{\theta}$ and $\tilde{\boldsymbol{\alpha}}$ denote generic values of phase and information sequence, respectively, which play the role of independent variables (continuous the first and discrete the second).

Substituting expression (6) of $s(t, \tilde{\boldsymbol{\alpha}})$ in (14), we obtain

$$
\begin{aligned}
\Lambda(\tilde{\theta}, \tilde{\boldsymbol{\alpha}})= & \operatorname{Re}\left\{e^{-j \tilde{\theta}} \sum_{n} \sum_{k=0}^{K-1} \tilde{a}_{k, n}^{*}\right. \\
& \left.\cdot \int_{-T_{0} / 2}^{T_{0} / 2} r(t) p_{k}(t-n T) d t\right\}
\end{aligned}
$$

where $\tilde{a}_{k, n}$ are obviously defined in terms of the information sequence $\tilde{\boldsymbol{\alpha}}$ [e.g., for MSK-type signals according to (10) and, for $K=2$, (11)]. For a sufficiently long observation interval $\left(T_{0} \rightarrow \infty\right)$, the integral in (15) may be interpreted as the output of a filter matched to the pulse $p_{k}(t)$ [i.e., with impulse response $\left.p_{k}(-t)\right]$. If we define

$$
r_{k, n} \triangleq \int_{-\infty}^{\infty} r(t) p_{k}(t-n T) d t=\left.r(t) \otimes p_{k}(-t)\right|_{t=n T}
$$

where $\otimes$ denotes convolution, the likelihood function (14) may be expressed as

$$
\Lambda(\tilde{\theta}, \tilde{\boldsymbol{\alpha}})=\operatorname{Re}\left\{e^{-j \tilde{\theta}} \sum_{n} \sum_{k=0}^{K-1} r_{k, n} \tilde{a}_{k, n}^{*}\right\} .
$$

A coherent ML detection receiver selects the information sequence according to

$$
\hat{\boldsymbol{\alpha}}=\arg \max _{\tilde{\boldsymbol{\alpha}}} \Lambda(\theta, \tilde{\boldsymbol{\alpha}})=\arg \max _{\tilde{\boldsymbol{\alpha}}}\left\{\sum_{n} \lambda_{n}(\theta, \tilde{\boldsymbol{\alpha}})\right\}
$$

where

$$
\lambda_{n}(\theta, \tilde{\boldsymbol{\alpha}}) \triangleq \operatorname{Re}\left\{e^{-j \theta} \sum_{k=0}^{K-1} r_{k, n} \tilde{a}_{k, n}^{*}\right\}
$$

and the variable $\tilde{\theta}$ takes on the value assumed by the channel phase shift $\theta \cdot \lambda_{n}(\theta, \tilde{\boldsymbol{\alpha}})$ in (19) may be interpreted as branch metrics in a properly defined trellis diagram. As a consequence, the maximization of (18) may be performed by means of a Viterbi algorithm. Considering a signal decomposition (6) by principal pulses only (i.e., $K \leq M-1$ ), it may be shown that, for multilevel formats $(M>2)$, the number of states of the relevant trellis diagram is $p$ (see the expression of the symbols $\left\{a_{k, n}\right\}$ corresponding to the principle pulses in [7]). This number of states must be compared to that of 
the optimal receiver $p M^{L-1}$. Beyond the state complexity reduction entailed by the simplified signal representation, in principle, a further reduction may be obtained by the technique of reduced state sequence estimation (RSSE) [16], first applied to CPM modulations in [4].

As an example for a quaternary CPM and assuming $K=3$ (signal represented by its principal pulses), the branch metrics depend only on symbols $a_{0, n}, a_{1, n}$ and $a_{2, n}$. Substituting (9) in (8), they may be expressed as (recall the assumption that $h$ and $2 h$ are not integers)

$$
\begin{aligned}
& a_{0, n}=e^{j h \pi \gamma_{n, 0}} e^{j 2 h \pi \gamma_{n, 1}} b_{0, n-1}^{(0)} b_{0, n-1}^{(1)} \\
& a_{1, n}=e^{j 2 h \pi \gamma_{n, 1}} b_{0, n-1}^{(0)} b_{0, n-1}^{(1)} \\
& a_{2, n}=e^{j h \pi \gamma_{n, 0}} b_{0, n-1}^{(0)} b_{0, n-1}^{(1)}
\end{aligned}
$$

and a state may be identified as

$$
\begin{aligned}
\mu_{n} & \triangleq b_{0, n-1}^{(0)} b_{0, n-1}^{(1)} \\
& =\exp \left\{j h \pi \sum_{m=0}^{n-1} \gamma_{m, 0}\right\} \exp \left\{j 2 h \pi \sum_{m=0}^{n-1} \gamma_{m, 1}\right\} \\
& =\exp \left\{j h \pi \sum_{m=0}^{n-1}\left(2 \gamma_{m, 1}+\gamma_{m, 0}\right)\right\} \\
& =\exp \left\{j h \pi \sum_{m=0}^{n-1} \alpha_{m}\right\} .
\end{aligned}
$$

This expression is similar to the phase state of a CPM signal - the total number of states is then $p$ [4], [14].

We now consider the problem of ML data-aided phase synchronization. The relevant likelihood function to be maximized is given by (17) in which $\tilde{\boldsymbol{\alpha}}$ equals the correct information symbol sequence $\alpha$. Accordingly, the ML data-aided phase estimate is

$$
\hat{\theta}=\arg \max _{\tilde{\theta}} \Lambda(\tilde{\theta}, \boldsymbol{\alpha})=\arg \left\{\sum_{n} \sum_{k=0}^{K-1} r_{k, n} a_{k, n}^{*}\right\} .
$$

This expression is a simple generalization of a well-known result valid for linear modulations, the only difference being the fact that a summation of terms is present (inner sum), which may be interpreted as contributions of each signal component of the extended Laurent representation. As a consequence, the standard derivation of ML data-aided phase synchronization schemes for linear modulations may be generalized in a straightforward fashion.

In order to obtain a solution in a recursive form, we may differentiate $\Lambda(\tilde{\theta}, \boldsymbol{\alpha})$ with respect to $\tilde{\theta}$, and perform the maximization by an iterative search through a gradient algorithm. The estimate $\hat{\theta}$ is the value of $\tilde{\theta}$ for which $d \Lambda(\tilde{\theta}, \boldsymbol{\alpha}) / d \tilde{\theta}$ vanishes. This value $\hat{\theta}$ may be obtained as the equilibrium of a recursive algorithm which models a second-order digital PLL according to

$$
\begin{aligned}
\xi_{n} & =\xi_{n-1}+(1+\alpha) e_{n}-e_{n-1} \\
\hat{\theta}_{n+1} & =\hat{\theta}_{n}+\gamma \xi_{n-d}
\end{aligned}
$$

where the error signal $e_{n}$ is

$$
e_{n} \triangleq \operatorname{Im}\left\{\sum_{k=0}^{K-1} x_{k, n} a_{k, n}^{*}\right\}
$$

having defined $x_{k, n} \triangleq e^{-j \hat{\theta}_{n}} r_{k, n}$. In (23), $\xi_{n}$ is the output of a first-order loop filter, $\hat{\theta}_{n}$ is the current estimate of $\theta, \alpha$ and $\gamma$ are the loop parameters, and we have introduced the delay $d$ which will be justified below. It is immediately apparent that the error signal is a sum of terms, each due to one of the signal components.

Besides possible applications of the above results in which either the phase reference may be considered known and a coherent detector implemented (pilot tone), or the information symbols may be considered known and a true data-aided ML phase synchronizer realized (training or pilot symbols), a common situation could be one in which simultaneous data detection and phase synchronization have to be performed. A typical solution to this specific problem of data detection without an explicit phase reference consists of utilizing a coherent detector which employs a phase reference obtained by a data-aided phase synchronizer driven by previous data decisions (decision-directed PLL). In this case, the branch metrics become

$$
\lambda_{n}\left(\hat{\theta}_{n}, \widetilde{\boldsymbol{\alpha}}\right)=\operatorname{Re}\left\{e^{-j \hat{\theta}_{n}} \sum_{k=0}^{K-1} r_{k, n} \tilde{a}_{k, n}^{*}\right\}
$$

and (24) is replaced by

$$
e_{n} \triangleq \operatorname{Im}\left\{\sum_{k=0}^{K-1} x_{k, n} \hat{a}_{k, n}^{*}\right\}
$$

where $\hat{a}_{k, n}$ are suitable data decisions.

As we have already noted, the receiver may contain a Viterbi processor which releases data decisions with a delay that may be large. This delay may be harmful to the tracking capabilities of a PLL. For this reason, we employ tentative low-delay decisions, made on the currently best survivor, to calculate the error $e_{n}$ in (26). Delay $d$ in (23) represents the number of steps backward, along the best survivor, necessary to make decisions which are reliable enough to direct the PLL. Large values of parameter $d$ yield more reliable decisions, but, at the same time, a degraded synchronizer response to rapid changes in the carrier phase. As a consequence, the value of $d$ has been optimized by simulation. The structure of this receiver which employs a decision-directed PLL is shown in Fig. 1, in which $D$ denotes the Viterbi algorithm detection depth.

\section{SYNCHRONIZER ANALYSIS AND OPTIMIZATION ALGORITHM}

In this section, we analyze the performance of the proposed data-aided ML synchronization schemes. This analysis is approximately valid for a decision-directed scheme under the realistic assumption of low error probability. 


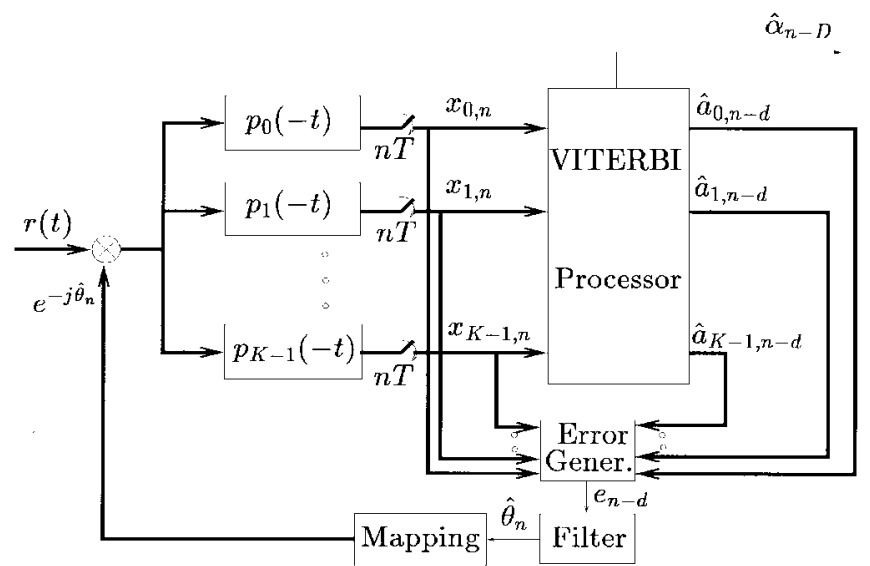

Fig. 1. Receiver and PLL structures.

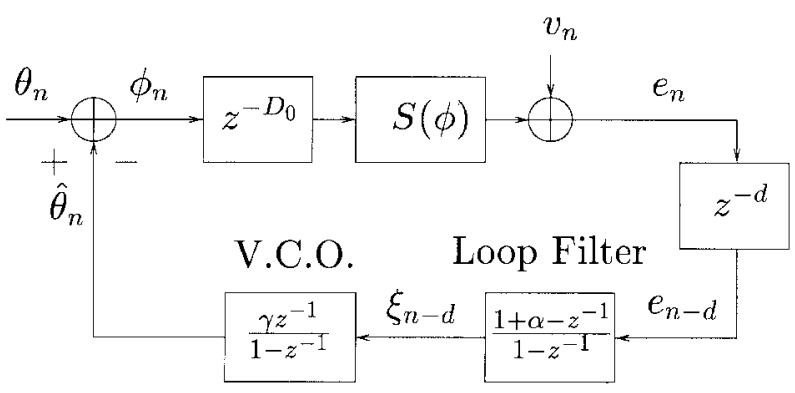

Fig. 2. Equivalent model of a PLL.

\section{A. Steady-State Analysis}

An equivalent model of a PLL is shown in Fig. 2 [13]. We define the phase error $\phi_{n} \triangleq \theta_{n}-\hat{\theta}_{n}$ and the parameter $D_{0}$ as the processing delay introduced by the filters matched to the pulses $p_{k}(t)$. The noise sequence $v_{n}$ depends on thermal noise and data symbols (see Appendix B). In the following, we indicate with $F(z)$ the transfer function of the cascade of the loop filter and voltage-controlled oscillator (VCO)

$$
F(z)=\frac{1+\alpha-z^{-1}}{1-z^{-1}} \cdot \frac{\gamma z^{-1}}{1-z^{-1}}=\frac{\gamma[(1+\alpha) z-1]}{(z-1)^{2}}
$$

which may easily be obtained by $Z$-transforming (23).

It is well-known that the $S$-curve of a PLL may be defined as

$$
S(\phi) \triangleq E\left\{e_{n} \mid \phi\right\}
$$

A linear equivalent model of a PLL is obtained substituting the $S$-curve with its linear approximation for $\phi=0$. By defining $\left.A \triangleq(d S(\phi) / d \phi)\right|_{\phi=0}$, the block $S(\phi)$ in Fig. 2 may be replaced by a linear element with input-output characteristic $A \phi$.

Assuming that all tentative decisions are correct, expressions for the $S$-curve and power spectral density of $v_{n}$ may be derived. These expressions are realistic models of the PLL behavior in the steady state and for a small phase jitter (i.e., $\phi \simeq 0$ ). For MSK-type modulations, an analytical derivation of the $S$-curve presented in Appendix A allows us to express
TABLE I

Numerator of $B_{\mathrm{EQ}} T$ for Given Values of $D_{0}+d$ and $A$

\begin{tabular}{c|c}
\hline \hline$D_{0}+d$ & $N(\alpha, \gamma)$ \\
\hline 0 & $2 \alpha+\gamma A(2+\alpha)$ \\
\hline 1 & $2 \alpha+\gamma A\left(2+\alpha+\alpha^{2}\right)+(\gamma A)^{2}(2+\alpha)$ \\
\hline \multirow{2}{*}{2} & $2 \alpha+2 \gamma A-\alpha \gamma A+2 \alpha^{2} \gamma A+2(\gamma A)^{2}-\alpha(\gamma A)^{2}-2 \alpha^{2}(\gamma A)^{2}-$ \\
& $-\alpha^{3}(\gamma A)^{2}-2(\gamma A)^{3}-\alpha(\gamma A)^{3}$ \\
\hline 3 & $2 \alpha+\left(2-\alpha+5 \alpha^{2}\right) \gamma A+\left(4-4 \alpha-2 \alpha^{2}-2 \alpha^{3}\right)(\gamma A)^{2}-$ \\
& $\left.-\left(2+\alpha+3 \alpha^{2}+3 \alpha^{3}+\alpha^{4}\right)(\gamma A)^{3}-(2+\alpha)(\gamma A)^{4}\right)$ \\
\hline \multirow{3}{*}{4} & $2 \alpha+\left(2-3 \alpha+8 \alpha^{2}\right) \gamma A+\left(4-8 \alpha-6 \alpha^{2}-8 \alpha^{3}\right)(\gamma A)^{2}+$ \\
& $+\left(3 \alpha-6-4 \alpha^{2}-4 \alpha^{3}-2 \alpha^{4}\right)(\gamma A)^{3}+(2+\alpha)(\gamma A)^{5}+$ \\
& $+\left(\alpha-2+4 \alpha^{2}+6 \alpha^{3}+4 \alpha^{4}+\alpha^{5}\right)(\gamma A)^{4}$ \\
\hline
\end{tabular}

TABLE II

Denominator of $B_{\mathrm{EQ}} T$ For Given Values of $D_{0}+d$ AND $A$

\begin{tabular}{c|c}
\hline \hline$D_{0}+d$ & $D(\alpha, \gamma)$ \\
\hline 0 & $2[4-\gamma A(2+\alpha)]$ \\
\hline 1 & $2\left[4(1-\alpha)-\gamma A\left(2+\alpha+\alpha^{2}\right)-(\gamma A)^{2}(2+\alpha)\right.$ \\
\hline 2 & $2\left(4-8 \alpha-6(\gamma A)+3 \alpha(\gamma A)-2 \alpha^{2}(\gamma A)-2(\gamma A)^{2}+\right.$ \\
& $\left.+\alpha(\gamma A)^{2}+2 \alpha^{2}(\gamma A)^{2}+\alpha^{3}(\gamma A)^{2}+2(\gamma A)^{3}+\alpha(\gamma A)^{3}\right)$ \\
\hline \multirow{3}{*}{3} & $8-24 \alpha+\left(-12+6 \alpha-22 \alpha^{2}\right)(\gamma A)+$ \\
& $+\left(-16+12 \alpha+4 \alpha^{2}+4 \alpha^{3}\right)(\gamma A)^{2}+$ \\
& $+\left(4+2 \alpha+6 \alpha^{2}+6 \alpha^{3}+2 \alpha^{4}\right)(\gamma A)^{3}+(4+2 \alpha)(\gamma A)^{4}$ \\
\hline \multirow{4}{*}{4} & $8-32 \alpha+\left(-20+30 \alpha-40 \alpha^{2}\right)(\gamma A)+$ \\
& $+\left(28 \alpha-16+20 \alpha^{2}+28 \alpha^{3}\right)(\gamma A)^{2}+$ \\
& $+\left(20-10 \alpha+8 \alpha^{2}+8 \alpha^{3}+4 \alpha^{4}\right)(\gamma A)^{3}+$ \\
& $+\left(4-2 \alpha-8 \alpha^{2}-12 \alpha^{3}-8 \alpha^{4}-2 \alpha^{5}\right)(\gamma A)^{4}-$ \\
\hline \hline
\end{tabular}

$A$ as

$$
A=\sqrt{2 E_{b}} \sum_{k=0}^{K-1} \int_{-\infty}^{+\infty}\left|p_{k}(t)\right|^{2} d t
$$

where $E_{b}$ is the received energy per information bit. This result has been confirmed by computer simulation. An expression of $S(\phi)$ for the general case of $M$-ary CPM signals may also be found in Appendix A.

In the steady state, the loop transfer function is

$$
\left.H(z) \triangleq \frac{\Phi(z)}{V(z)}\right|_{\theta_{n} \equiv \theta_{0}}=-\frac{F(z) z^{D_{0}}}{z^{D_{0}+d}+A F(z)}
$$

where $\Phi(z)$ and $V(z)$ are the $Z$-transform of $\phi_{n}$ and $v_{n}$, respectively. This transfer function allows us to evaluate the one-sided noise-equivalent bandwidth of the loop $B_{\mathrm{EQ}}$ defined as (see [17, p. 187])

$$
\begin{aligned}
B_{\mathrm{EQ}} T & \triangleq \frac{1}{2|H(1)|^{2}} \int_{-1 / 2}^{1 / 2}\left|H\left(e^{j 2 \pi f T}\right)\right|^{2} d(f T) \\
& =\frac{1}{j 4 \pi|H(1)|^{2}} \oint \frac{H(z) H\left(z^{-1}\right)}{z} d z
\end{aligned}
$$

This second integral may be analytically evaluated using Cauchy's residue theorem. The resulting expression of $B_{\mathrm{EQ}} T$ may be put in the rational form

$$
B_{\mathrm{EQ}} T=\frac{N(\alpha, \gamma)}{D(\alpha, \gamma)}
$$

where the polynomials $N(\alpha, \gamma)$ and $D(\alpha, \gamma)$ are given in Tables I and II for some values of $D_{0}+d$ (from (30) and (31), it is clear that $B_{\mathrm{EQ}} T$ depends on $D_{0}$ and $d$ through their sum $D_{0}+d$ ). An extended list may be found in [18]. 
The evaluation of the power spectral density $W_{v}(f)$ of $v_{n}$ described for TFM in Appendix B allows us to conclude that $W_{v}(f)$ is: 1) practically independent of the data sequence, and 2) nearly constant in the frequency range of interest, with $W_{v}(f) \simeq W_{v}(0) \simeq N_{0}$. Therefore, for TFM, the phase error variance may be expressed as

$$
\sigma_{\phi}^{2}=W_{v}(0) \int_{-1 / 2}^{1 / 2}\left|H\left(e^{j 2 \pi f T}\right)\right|^{2} d(f T)=\frac{N_{0}}{E_{b}} B_{\mathrm{EQ}} T
$$

which coincides with the modified Cramer-Rao lower bound (MCRB) [19]. This fact confirms the satisfactory quality of the estimation process.

The analysis has been completed by simulations, taking into account the presence of phase jitter and measuring the mean time between phase slips. Simulation results are discussed in Section V.

\section{B. Optimization Algorithm}

Based on the results in Section IV-A, we are in the position to select the loop parameters $\alpha$ and $\gamma$ in order to specify the value of the loop noise-equivalent bandwidth $B_{\mathrm{EQ}} T$ for a given value of the delay parameter $D_{0}+d$. Since, by fixing the value of $B_{\mathrm{EQ}} T$, we have one equation (32) and two unknowns $\alpha$ and $\gamma$, we exploit this degree of freedom to optimize the transient response of the PLL in the presence of instabilities of up- and down-conversion oscillators. Specifically, we are concerned with short-term instabilities which manifest themselves as random rapid variations of the oscillation frequency due to the thermal or mechanical stress of the components or mechanical structures [12]. These transient phenomena of short duration (comparable with the bit interval) and rare occurrence over a long period may be properly modeled as phase-continuous frequency offset steps.

The parameter which affects the system performance is the relative frequency offset $\nu_{0} T$ (normalized to the symbol frequency $1 / T$ ), where $\nu_{0}$ is the absolute frequency error. As a consequence, this phenomenon may cause quite a serious problem in low-data-rate systems. Furthermore, the higher the carrier frequency, the larger is the frequency offset (for a given oscillator stability). When such a type of instability occurs, the receiver should possibly continue to detect information symbols correctly. In this scenario, the loop parameters must be optimized for given values of $B_{\mathrm{EQ}} T$ and $D_{0}+d$ in order to maximize the amplitude of frequency steps which do not induce data decision errors.

Neglecting thermal noise, we assume that the PLL is in its linear operating condition because we require the absence of data detection errors (lock-in condition). From results in the previous section, in the absence of thermal noise, we may consider $W_{v}(f)=0$. As a consequence, we may define the following transfer function:

$$
\left.M(z) \triangleq \frac{\Phi(z)}{\Theta(z)}\right|_{V(z)=0}=\frac{z^{D_{0}+d}}{z^{D_{0}+d}+A F(z)}
$$

where $\Theta(z)$ is the $Z$-transform of $\theta_{n}$. If the incoming signal is affected by a frequency offset step beginning at time $t=0$ (i.e., a ramp in the channel phase), one has

$$
\Theta(z)=\frac{2 \pi \nu_{0} T z}{(z-1)^{2}}
$$

where $\nu_{0}$ is the frequency offset amplitude. The $Z$-transform of the phase error evolution $\phi_{n}$ induced by the frequency offset step is then expressed by

$$
\Phi(z)=M(z) \frac{2 \pi \nu_{0} T z}{(z-1)^{2}} .
$$

For each value of $B_{\mathrm{EQ}} T$ and $D_{0}+d$, our objective is to find the loop parameters $\alpha$ and $\gamma$ which minimize the energy associated with the transient phase error $\phi_{n}$ following a frequency step. This energy may be expressed as [17]

$$
\mathcal{E}(\alpha, \gamma) \triangleq \sum_{n}\left|\phi_{n}\right|^{2}=\frac{1}{j 2 \pi} \oint \frac{\Phi(z) \Phi\left(z^{-1}\right)}{z} d z .
$$

We are then faced with a constrained optimization problem in which $\mathcal{E}(\alpha, \gamma)$ must be minimized subject to a constraint on the noise-equivalent bandwidth $B_{\mathrm{EQ}} T$. Using a Lagrange multiplier, we minimize the function

$$
f(\alpha, \gamma, \lambda) \triangleq \mathcal{E}(\alpha, \gamma)+\lambda\left[\frac{N(\alpha, \gamma)}{D(\alpha, \gamma)}-B_{\mathrm{EQ}} T\right]
$$

for each value of $B_{\mathrm{EQ}} T$ and $D_{0}+d$ in which we are interested. The resulting optimal values of the loop parameters $\alpha$ and $\gamma$ versus $B_{\mathrm{EQ}} T$ and $D_{0}+d$ may be found in [18].

A final optimization step on the tentative decision delay $d$ has been based on simulation results.

\section{NUMERICAL RESUltS}

In this section, we consider a TFM signal [15] which is well known to belong to the class of MSK-type signals (i.e., $M=2$ and $h=0.5$ ). In this case, the frequency pulse has Fourier transform

$$
G(f)=\mathcal{F}[g(t)]= \begin{cases}\frac{1}{2} \frac{\cos ^{2}(\pi f T)}{\operatorname{sinc}(f T)}, & \text { for }|f| \leq \frac{1}{2 T} \\ 0, & \text { elsewhere }\end{cases}
$$

where $\operatorname{sinc}(x) \triangleq \sin (\pi x) / \pi x$. In the TFM case, the frequency pulse may be truncated with good approximation to an interval of duration $L T$ with $L \geq 4$. We assume $L=5$ in our numerical results. Therefore, the length of $p_{0}(t)$ is of $(L+1)=$ 6 symbol intervals. However, we truncated the length of the matched filter responses to 4 symbol intervals in order to limit the delay $D_{0}$ as much as possible. Negligible degradation in the BER has been observed as a consequence of this truncation. The resulting value of delay is $D_{0}=2$.

Two receivers have been considered for the TFM format. The first receiver is composed of two filters matched to the pulses $p_{0}(t)$ and $p_{1}(t)$ for each signal dimension (i.e., the real and imaginary parts of the baseband-equivalent received signal) and a four-state Viterbi processor according to [8]. For TFM, this receiver is practically optimal because the signal power contained in the first two signal components is with very good approximation equal to the total power [18]. The value of $K$ in (26) is set to 2 because the receiver already 
contains the two filters needed for the generation of $e_{n}$, and no performance improvement has been observed for $K>2$. The second receiver is a symbol-by-symbol one composed of a filter (for each signal dimension) matched to the pulse $p_{0}(t)$ (we assume $K=1$ ), a decision feedback equalizer which minimizes the MSE taking into account intersymbol interference and noise (with the feedforward filter composed of two taps and a one tap feedback filter), and a threshold detector. This receiver exhibits a performance degradation of only $0.15 \mathrm{~dB}$ with respect to the optimal receiver at a bit-error rate (BER) of $10^{-4}$.

We also consider a quaternary 2RC modulation with $h=$ 0.25 and frequency pulse

$$
g(t)= \begin{cases}\frac{1}{4 T}\left(1-\cos \frac{\pi t}{T}\right), & \text { for } 0 \leq t \leq 2 T \\ 0, & \text { elsewhere }\end{cases}
$$

In this case, the number of principal pulses is $K=3$, as already pointed out in Section II. In principle, the necessary number of matched filters would be $K=3$. However, due to the similarity of pulses $p_{1}(t)$ and $p_{2}(t)$, negligible degradation is implied by the use of an average filter for both pulses. As a consequence, a total number of two filters for each signal dimension is required ${ }^{1}$. The considered receiver is based on a four-state Viterbi processor. In this case, the length of $p_{0}(t)$ is $(L+1)=3$, which corresponds to a delay $D_{0}=1$ of the corresponding matched filter because of the assumed FIR (finite impulse response) structure with 2 samples per symbol interval (this assumption was also used for TFM).

The optimization algorithm described in Section IV has been used to determine the loop parameter values for each value of $B_{\mathrm{EQ}} T$ and $d$.

$S$ curves normalized to $\sqrt{2 E_{S}}$ have been obtained by computer simulation, and are shown in Fig. 3 for quaternary 2RC modulation and various values of $K$ and signal-to-noise ratio. This figure shows that, if we consider only the first signal component, the PLL performance may experience noticeable degradations. However, no improvement can be obtained by considering more than three components. Fig. 3 also shows the $S$-curve for $K=3$ and various signal-to-noise ratios. $S$ curves for TFM, not reported here for lack of space, practically coincide for $K=1$ and $K=2$, suggesting that only a negligible performance improvement can be obtained by assuming a more refined signal representation (i.e., $K \geq$ 2). These conclusions are also valid for other MSK-type modulations we considered.

In Fig. 4, we show, for TFM modulation, the phase error variance measured by computer simulation and compared with the analytical result (33). The theoretical result is found to be in good agreement with simulation.

The analysis has been extended with simulations in the presence of phase jitter. For this purpose, we assume that the phase $\theta$ of the received signal is modeled as a discrete-time Wiener process

$$
\theta_{n+1}=\theta_{n}+\Delta_{n}
$$

\footnotetext{
${ }^{1}$ The authors thank M. Morelli for suggesting this possibility of further complexity reduction.
}

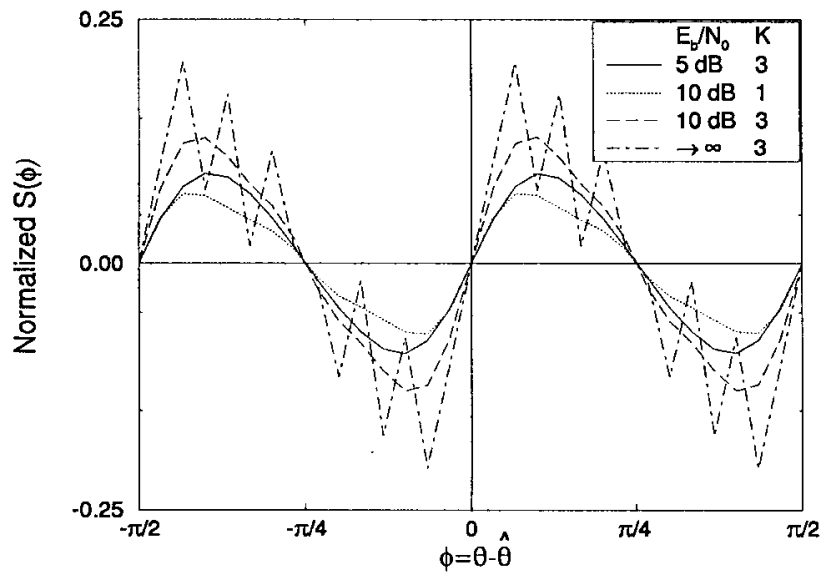

Fig. 3. Quaternary 2RC modulation: $S$-curve for various values of $E_{b} / N_{0}$ and $K$.

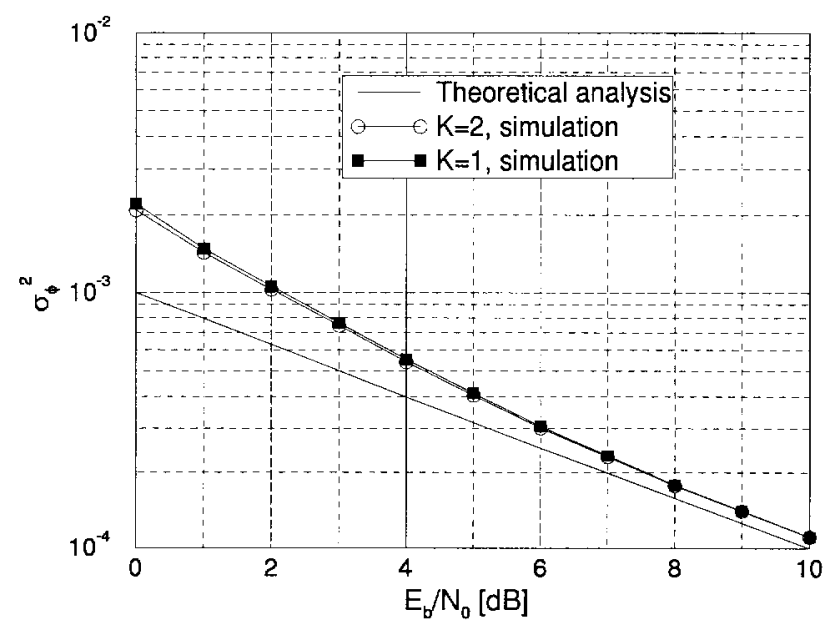

Fig. 4. TFM modulation: phase error variance versus $E_{b} / N_{0}$ for $B_{\mathrm{EQ}} T=10^{-3}$.

where $\Delta_{n}$ are zero-mean independent Gaussian random variables with variance $\sigma_{\Delta}^{2}$. This model is frequently used for its good agreement with experimental data [20]. We point out that, for each signal-to-noise ratio, an optimal value of the noise-equivalent bandwidth exists, which minimizes the biterror probability. For increasing values of $B_{\mathrm{EQ}}$, the noise $v_{n}$ yields a performance degradation; on the contrary, the PLL capacity to track the phase jitter without losing lock improves.

Fig. 5 shows the BER versus $E_{b} / N_{0}$ of optimal and simplified coherent four-state Viterbi receivers for TFM modulation. No performance degradation due to the reduced number of states in the simplified receiver is observed. This figure also shows the performance of four-state Viterbi receivers employing a decision-directed PLL in the presence of phase jitter for different values of $\sigma_{\Delta}$. In this condition, the system with $d=0$ exhibits the best performance. A similar analysis was performed for the considered quaternary $2 \mathrm{RC}$ modulation. The relevant results are shown in Fig. 6. First, we note that a minor performance degradation of about $0.15 \mathrm{~dB}$ is exhibited by the simplified four-state Viterbi coherent receiver with respect to the optimal one (at a BER of $10^{-4}$ ). In the presence of phase jitter, the performance of the considered simplified receiver with decision directed PLL is optimized for a tentative 


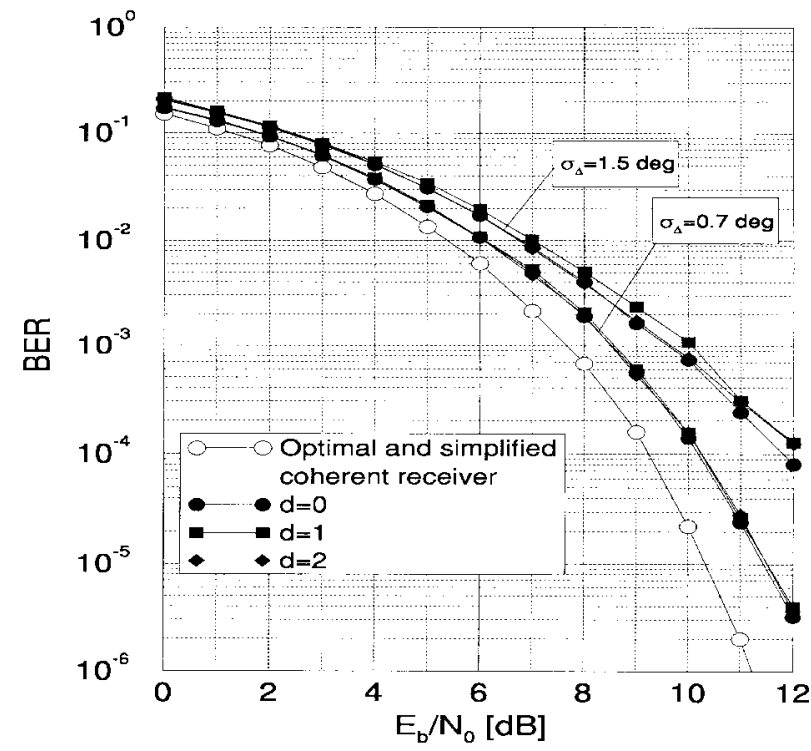

Fig. 5. TFM modulation: BER versus $E_{b} / N_{0}$ for coherent and PLL-based Viterbi receivers with $D_{0}=2$, various values of $d$, and phase jitter with $\sigma_{\Delta}=0.7,1.5^{\circ}$.

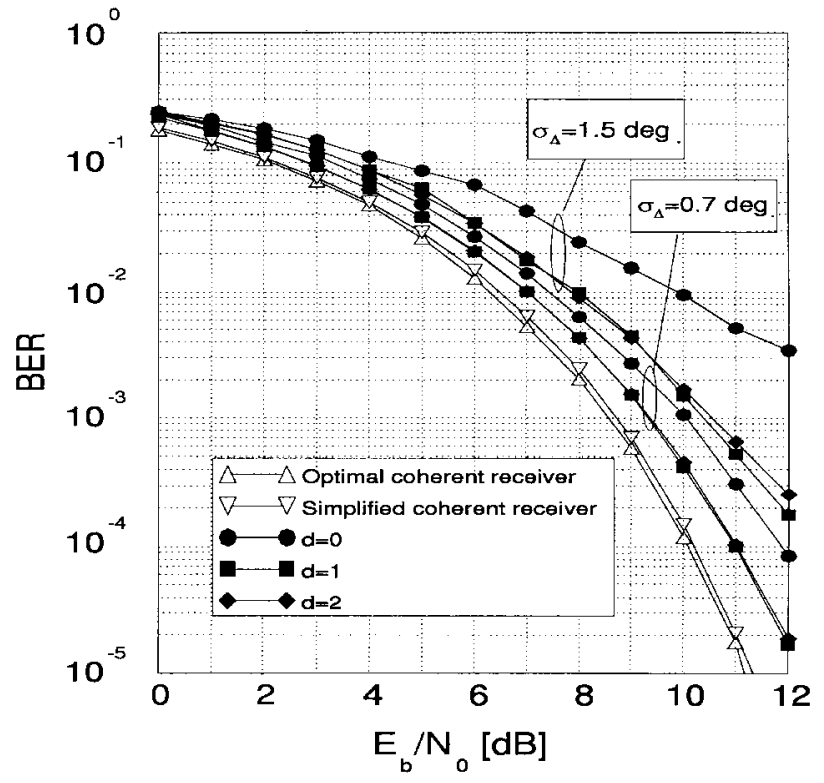

Fig. 6. Quaternary 2RC modulation: BER versus $E_{b} / N_{0}$ for coherent and PLL-based Viterbi receivers with $D_{0}=1$, various values of $d$, and phase jitter with $\sigma_{\Delta}=0.7,1.5^{\circ}$

decision delay $d=1$. In both Figs. 5 and 6 , the value of $B_{\mathrm{EQ}} T$ has been optimized by simulation for each value of $E_{b} / N_{0}$. However, we have verified that this optimization is not critical-the use of an average value of $B_{\mathrm{EQ}} T$ would entail negligible degradation.

A simulation analysis of the acquisition time has also been performed. The results of this analysis show that in worst cases (initial phase errors corresponding to zeros with negative slope of the $S$-curve) the acquisition time is on the order of a few tens of symbols only.

The mean time to slip (MTS) has also been analyzed. If the incoming phase is $\theta=\theta_{0}$ constant, the PLL locks around the equilibrium points $\hat{\theta}=\theta_{0}+(2 \pi k / M)$ with fluctuations caused

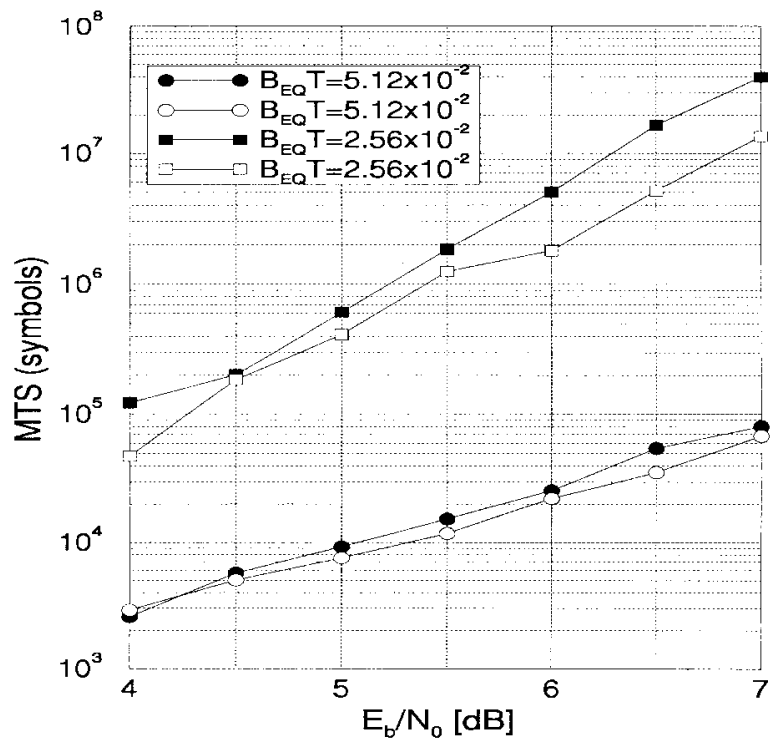

Fig. 7. TFM modulation: MTS versus $E_{b} / N_{0}$ for $D_{0}=2$ and various values of $B_{\mathrm{EQ}} T$. Receivers considered: four-state Viterbi receiver (black marks) with $d=0$ and symbol-by-symbol receiver (white marks).

by the noise $v_{n}$. A phase slip occurs when these fluctuations move the PLL toward another equilibrium point, producing an error (or a few) on data decisions. The MTS is the average time between two successive phase slips. Fig. 7 shows, for TFM modulation, the MTS versus $E_{b} / N_{0}$ for different values of $B_{\mathrm{EQ}} T .50$ phase slip occurrences have been used to measure each value of MTS. As may be seen, the four-state Viterbi and symbol-by-symbol receivers perform identically.

Fig. 8 shows the amplitude of maximum frequency steps $\nu_{\max } T$ (normalized to the symbol frequency) versus $B_{\mathrm{EQ}} T$, which the considered PLL can track without any decision errors. These results are relative to $2 \mathrm{RC}$ modulation, and are based on 100 independent simulation runs for each value of $B_{\mathrm{EQ}} T$ and $d$. This simulation has been performed with $E_{b} / N_{0}=30 \mathrm{~dB}$ in order to separate the effects of thermal noise from those due to the frequency step. As we can see, the proposed optimization algorithm allows the PLL to perform as well as an idealized PLL directed by correct decisions (curve marked "known data"). An increase in $B_{\mathrm{EQ}} T$ makes the PLL able to cope with larger frequency steps on the order of $10^{-3}$. Similar values of frequency step amplitude have been observed for TFM, in which case the behavior of the Viterbi and symbol-by-symbol receivers is practically identical.

\section{CONCLUSIONS}

In this paper, a class of reduced-complexity detection and phase synchronization schemes for multilevel CPM signals has been presented, based on ML estimation and extended Laurent representation. This representation is a powerful tool for the design of reduced-complexity receivers.

We have first extended the results by Kaleh [8] to the case of multilevel formats. The complexity of the resulting detection schemes, expressed in terms of number of matched filters and states of a Viterbi algorithm, is significantly reduced with respect to that of optimal coherent receivers with negligible 


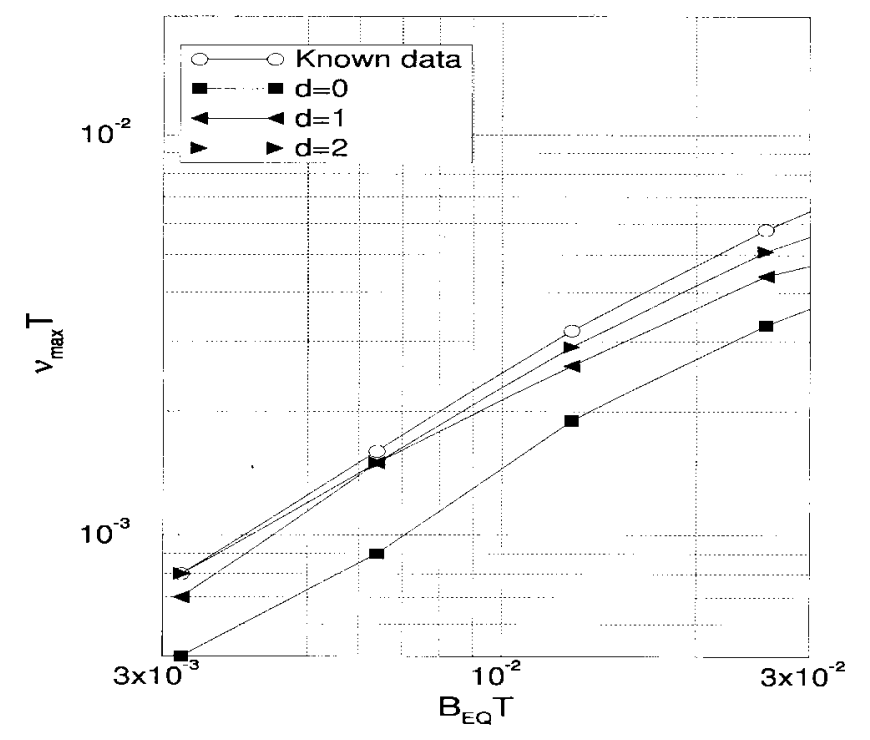

Fig. 8. Quaternary 2RC modulation: $\nu_{\max } T$ versus $B_{\mathrm{EQ}} T$ for $E_{b} / N_{0}=30 \mathrm{~dB}, K=3$, and $D_{0}=1$.

performance loss. As an example for a quaternary 2RC modulation with $h=0.25$, a four-state Viterbi receiver is only about 0.1-0.2 dB worse than an optimal receiver with 16 states.

The structure of the proposed synchronization schemes is an extension of a classical digital PLL for linear modulations. The additional complexity necessary for its implementation is moderate because this subsystem uses the same matched filters necessary for data detection.

A theoretical performance analysis of the proposed synchronization schemes has been performed. Computer simulation has been used to confirm the theoretical results and extend the analysis by considering robustness to phase jitter, mean time between phase slips, and acquisition time. Based on this analysis, a small number of signal components must be taken into account in order to practically attain optimal performance.

A loop parameter optimization algorithm has been proposed to effectively cope with the effects of frequency instabilities. The resulting synchronization scheme is capable of tracking, with no detection errors, instantaneous frequency offset steps whose amplitude, normalized to the signaling frequency, is on the order of $10^{-3}$. The optimized PLL performs as well as an idealized PLL directed by correct decisions.

\section{APPENDIX A}

In this Appendix, we compute the $S$-curve $S(\phi)$ under the assumption of correct decisions, i.e., $\hat{a}_{k, n}=a_{k, n}$. From (28) and (24), one has

$$
S(\phi)=E\left\{e_{n} \mid \phi\right\}=E\left\{\operatorname{Im}\left[\sum_{k=0}^{K-1} x_{k, n} a_{k, n}^{*}\right] \mid \phi\right\}
$$

where $x_{k, n}=r_{k, n} e^{-j \hat{\theta}}$. Substituting (6) in (13) and the result in (16), we may express

$$
\begin{aligned}
x_{k, n} a_{k, n}^{*}= & \sqrt{2 E_{b}}\left[\sum_{m=0}^{K-1} \sum_{i} a_{m, i} a_{k, n}^{*} \rho_{m, k}[(n-i) T]\right] e^{j \phi} \\
& +n_{k}(n T) a_{k, n}^{*}
\end{aligned}
$$

where

$$
\begin{gathered}
\rho_{m, k}(t) \triangleq p_{m}(t) \otimes p_{k}(-t) \\
n_{k}(t) \triangleq w(t) \otimes p_{k}(-t) .
\end{gathered}
$$

Using the independence between noise and information symbols, we obtain

$$
\begin{aligned}
S(\phi)= & \sqrt{2 E_{S}} \operatorname{Im}\left\{\sum_{k=0}^{K-1} \sum_{m=0}^{K-1} \sum_{i} R_{m, k}(n-i)\right. \\
& \left.\cdot \rho_{m, k}[(n-i) T] e^{j \phi}\right\} .
\end{aligned}
$$

In the case of MSK-type modulations, using (12), one has

$$
\begin{aligned}
S(\phi) & =\sqrt{2 E_{b}}\left\{\sum_{k=0}^{K-1} \rho_{k, k}(0)\right\} \sin \phi \\
& =\sqrt{2 E_{b}}\left\{\sum_{k=0}^{K-1} \int_{-\infty}^{+\infty}\left|p_{k}(t)\right|^{2} d t\right\} \sin \phi .
\end{aligned}
$$

The derivative for $\phi=0$ yields (29).

\section{APPENDIX B}

In this Appendix, we approximately evaluate the power spectral density of $v_{n}$ for the case of TFM modulation. The same procedure may be applied to different MSK-type modulations and extended to multilevel formats.

To approximately evaluate the power spectral density $W_{v}(f)$ in the steady state, we write $v_{n}$ in the form (see Fig. 2)

$$
v_{n}=e_{n}-S\left[\theta-\hat{\theta}_{n}\right]=\operatorname{Im}\left\{\sum_{k=0}^{K-1} x_{k, n} a_{k, n}^{*}\right\}-S(\phi)
$$

which holds under the assumption of a constant $\hat{\theta}_{n} \simeq \theta(\phi \simeq$ 0 ) based on the fact that $\hat{\theta}_{n}$ varies slowly compared to $e_{n}$ because of the limited values of $B_{E Q} T$. Using (A2), we obtain (again under the assumption of correct decisions)

$$
\begin{aligned}
e_{n}= & \operatorname{Im}\left\{\sum_{k=0}^{K-1} x_{k, n} a_{k, n}^{*}\right\} \\
= & \sqrt{2 E_{b}} \sum_{k=0}^{K-1} \sum_{m=0}^{K-1} \sum_{i} \operatorname{Im}\left\{a_{m, i} a_{k, n}^{*} e^{j \phi}\right\} \rho_{m, k}[(n-i) T] \\
& +\operatorname{Im}\left\{\sum_{k=0}^{K-1} n_{k}(n T) a_{k, n}^{*} e^{-j \hat{\theta}}\right\} .
\end{aligned}
$$

Introducing some approximations, it is possible to obtain an analytical expression of $v_{n}$. To this end, we consider only the first two signal components neglecting those with less power, i.e., we let $K=2$ in (B2). The desired expression of $v_{n}$ is obtained by subtracting (A5) to (B2), i.e., by eliminating from (B2) the terms for $k=0$ and $i=n$ in the first term of the outer sum and the terms for $k=1$ and $i=n$ in the second term of the outer sum. We also neglect $\rho_{1,1}(t)$ whose maximum value 
is the energy of the second signal component, and consider only the significant samples of $\rho_{0,0}(t)$ and $\rho_{1,0}(t)$. We use the properties $\rho_{0,0}(-t)=\rho_{0,0}(t), \rho_{0,1}(-t)=\rho_{1,0}(t),(10)$ and (11). After some algebraic manipulations, we obtain, for $K=2$,

$$
\begin{aligned}
v_{n}= & \operatorname{Im}\left\{\left[n_{0}(n T) a_{0, n}^{*}+n_{1}(n T) a_{1, n}^{*}\right] e^{-j \hat{\theta}}\right\} \\
& +\sqrt{2 E_{b}}\left[\sin \phi\left(a_{0, n-2} a_{0, n}^{*}+a_{0, n+2} a_{0, n}^{*}\right) \rho_{0,0}(2 T)\right. \\
& \left.-j \cos \phi\left(a_{0, n-1} a_{0, n}^{*}+a_{0, n+1} a_{0, n}^{*}\right) \rho_{0,0}(T)\right] \\
& +\sqrt{2 E_{b}}\left[\sin \phi\left(a_{1, n+1} a_{0, n}^{*}+a_{0, n-1} a_{1, n}^{*}\right) \rho_{1,0}(-T)\right. \\
& \left.-j \cos \phi\left(a_{1, n+2} a_{0, n}^{*}+a_{0, n-2} a_{1, n}^{*}\right) \rho_{1,0}(-2 T)\right] .(\mathrm{B} 3)
\end{aligned}
$$

This expression allows us to compute the autocorrelation sequence of $v_{n}$. Taking into account that the variance of the imaginary part of $n_{k}(t)$ is $N_{0} \rho_{k, k}(0)$, the autocorrelation sequence $R_{v}(n)$ may be approximated as

$$
\begin{aligned}
& R_{v}(0) \simeq N_{0}\left[\rho_{0,0}(0)+\rho_{1,1}(0)\right] \\
&+4 E_{b} \cos ^{2} \phi\left[\rho_{0,0}^{2}(T)+\rho_{1,0}^{2}(-2 T)\right] \\
&+4 E_{b} \sin ^{2} \phi\left[\rho_{0,0}^{2}(2 T)+\rho_{1,0}^{2}(-T)\right] \\
& R_{v}(1) \simeq 2 E_{b}\left[-\cos ^{2} \phi \rho_{0,0}^{2}(T)+\sin ^{2} \phi \rho_{1,0}^{2}(-T)\right] \\
& R_{v}(2) \simeq 2 E_{b}\left[\sin ^{2} \phi \rho_{0,0}^{2}(2 T)-\cos ^{2} \phi \rho_{1,0}^{2}(-2 T)\right] \\
& R_{v}(k) \simeq 0, \quad \text { for } k>2 .
\end{aligned}
$$

Finally, the power spectral density $W_{v}(f)$ is computed by Fourier transformation, and has the expression

$$
\begin{gathered}
W_{v}(f) \simeq R_{v}(0)+2 R_{v}(1) \cos 2 \pi f T \\
+2 R_{v}(2) \cos 4 \pi f T .
\end{gathered}
$$

The value for $f=0$ (noting that $\rho_{0,0}(0)+\rho_{1,1}(0) \simeq 1$ ) is

$$
W_{v}(0) \simeq N_{0}+8 E_{b} \sin ^{2} \phi\left[\rho_{0,0}^{2}(2 T)+\rho_{1,0}^{2}(-T)\right]
$$

In the steady state $(\phi \simeq 0)$, the dominant term is represented by thermal noise.

\section{ACKNOWLEDGMENT}

The authors acknowledge the contribution of M. Quarantelli in developing part of the computer programs.

\section{REFERENCES}

[1] J. B. Anderson, T. Aulin, and C.-E. Sundberg, Digital Phase Modulation. New York: Plenum, 1986.

[2] A. Svensson, "Reduced state sequence detection of partial response continuous phase modulation," Proc. Inst. Elect. Eng., vol. 138, pt. I, pp. 256-268, Aug. 1991.

[3] S. J. Simmons and P. H. Wittke, "Low complexity decoders for constant envelope digital modulations," IEEE Trans. Commun., vol. COM-31, pp. 1273-1280, Dec. 1983.

[4] J. Huber and W. Liu, "An alternative approach to reduced-complexity CPM-receivers," IEEE J. Select. Areas Commun., vol. SAC-7, pp. 1437-1449, Dec. 1989.
[5] __ "Data-aided synchronization of coherent CPM-receivers," IEEE Trans. Commun., vol. 40, pp. 178-189, Jan. 1990.

[6] P. A. Laurent, "Exact and approximate construction of digital phase modulations by superposition of amplitude modulated pulses (AMP)," IEEE Trans. Commun., vol. COM-34, pp. 150-160, Feb. 1986.

[7] U. Mengali and M. Morelli, "Decomposition of $M$-ary CPM signals into PAM waveforms," IEEE Trans. Inform. Theory, vol. IT-41, pp. 1265-1275, Sept. 1995.

[8] G. K. Kaleh, "Simple coherent receivers for partial response continuous phase modulation," IEEE J. Select. Areas Commun., vol. 7, pp. 1427-1436, Dec. 1989.

[9] O. Andrisano and M. Chiani, "The first Nyquist criterion applied to coherent receiver design for generalized MSK signals," IEEE Trans. Commun., vol. 42, pp. 449-457, Feb./Mar./Apr. 1994.

[10] A. N. D'Andrea, A. Ginesi, and U. Mengali, "Frequency detectors for CPM signals," IEEE Trans. Commun., vol. 43, pp. 1828-1837, Feb./Mar./Apr. 1995.

[11] G. K. Kaleh, "Carrier phase recovery for partial response continuous phase modulation with index 0.5," in Communication, Control, and Signal Processing, E. Arikan, Ed. Amsterdam, The Netherlands: Elsevier, 1990.

[12] B. Baccetti and A. Giavarini, "Microwave technology in modern fixed point-to-point radio systems," in Proc. 25th EUMC (European Microwave Conf.), Bologna, Italy, Sept. 1995.

[13] W. C. Lindsey and C. M. Chie, "A survey of digital phase-locked loops," Proc. IEEE, vol. 69, pp. 410-431, Apr. 1981.

[14] B. E. Rimoldi, "A decomposition approach to CPM," IEEE Trans. Inform. Theory, vol. 34, pp. 260-270, Mar. 1988.

[15] F. de Jager and C. B. Dekker, "Tamed frequency modulation, A novel method to achieve spectrum economy in digital transmission," IEEE Trans. Commun., vol. COM-26, pp. 534-542, May 1978.

[16] M. V. Eyuboğlu and S. U. H. Qureshi, "Reduced-state sequence estimation with set partitioning and decision feedback," IEEE Trans. Commun., vol. 36, pp. 13-20, Jan. 1988.

[17] A. V. Oppenheim, R. W. Schafer, Discrete-Time Signal Processing. Englewood Cliffs, NJ: Prentice-Hall, 1989.

[18] Research cooperation between the Dipartimento di Ingegneria dell'Informazione, Università di Parma, and Italtel S.p.A., Tech. Rep. 2, Part II-CPM Systems (in Italian), Jan. 1996.

[19] A. N. D'Andrea, U. Mengali and R. Reggiannini, "The modified Cramer-Rao bound and its application to synchronization problems," IEEE Trans. Commun., vol. COM-42, pp. 1391-1399, Feb./Mar./Apr. 1994.

[20] F. M. Gardner, Phaselock Techniques, 2nd ed. New York: Wiley, 1979

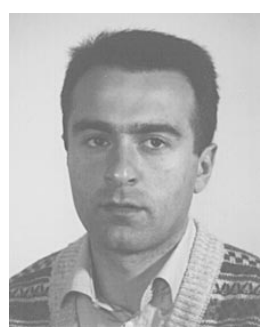

Giulio Colavolpe (S'97) was born in Cosenza, Italy, in 1969. He received the Dr.Ing. degree in telecommunications engineering (cum laude) from the University of Pisa, Italy, in 1994. He is currently working toward the Ph.D. degree in information technology at the University of Parma, Italy.

His main research interests include digital transmission theory, channel coding, and signal processing.

Riccardo Raheli (M'87) was born in Alezio (Lecce), Italy, in 1957. He received the Dr.Ing. degree (Laurea) from the University of Pisa, Italy, in 1983, the M.Sc. degree from the University of Massachusetts, Amherst, in 1986, and the Doctoral degree (Perfezionamento) from the Scuola Superiore di Studi Universitari e di Perfezionamento, Pisa, Italy, in 1987, all in electrical engineering.

From 1986 to 1988, he was with the Radio System Division of Siemens Telecomunicazioni, Milan, Italy. From 1988 to 1991, he was a faculty member at the Scuola Superiore di Studi Universitari e di Perfezionamento S. Anna, Pisa, Italy. In 1990, he was a Visiting Assistant Professor at the University of Southern California, Los Angeles. Since 1991, he has been a faculty member at the University of Parma, Italy. His current research interests include digital transmission theory, sequence detection techniques, signal processing, and adaptive algorithms for communications. 